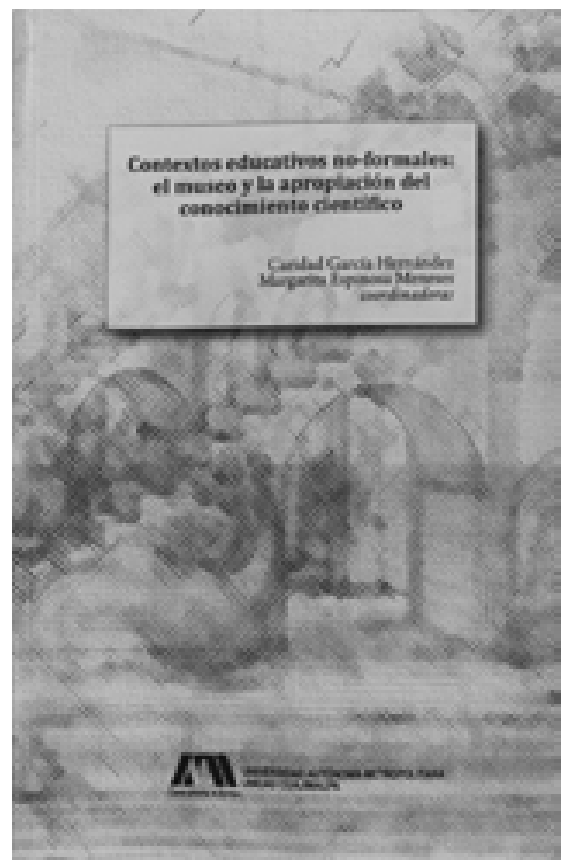

FIGURA 1. Portada del libro Contextos educativos no formales: el museo y la apropiación del conocimiento científico, de Caridad García Hernández y Margarita Espinosa Meneses (coords.), Universidad Autónoma Metropolitana-Unidad Cuajimalpa (UAM-C), México, 2015, ISBN: 978-6072803701.

\section{Tomar el conocimiento para sí: reseña de Contextos educativos no formales: el museo y la apropiación del conocimiento científico}

Taking Knowledge for Yourself: Book Review of Non-formal Educational Contexts: Museums and the Appropriation of Scientific Knowledge

\section{Norma Angélica Avila Meléndez}

Instituto de Educación Media Superior del Distrito Federal (IEMS-DF), México norma.avila@iems.edu.mx

\section{Resumen}

Esta RESEÑA del libro intitulado Contextos educativos no formales: el museo y la apropiación del conocimiento científico (García y Espinosa 2015) resalta un trabajo colectivo que articula evidencias empíricas y reflexiones de corte epistémico acerca de la apropiación del conocimiento científico en la educación no formal. Este tema es de especial interés para los profesionales atentos a las relaciones entre ciencia y sociedad, ya que propicia tres aspectos: en primer lugar, que los educadores revisen las teorías de los aprendizajes subyacentes a sus prácticas; en segundo término, que los museólogos conozcan una aproximación científica al conocimiento apropiado, y por último, que los investigadores ponderen la distribución social del conocimiento científico y el papel que los museos alcanzan a desempeñar en el contexto nacional.

\section{Palabras clave}

apropiación del conocimiento científico; cibermuseo; educación no formal; museos y conocimiento apropiado; México

\section{Abstract}

This BOOK REVIEW of Non-formal Educational Contexts: Museums and the Appropriation of Scientific Knowledge (García and Espinosa 2015) highlights a collective work that presents empirical evidence and epistemic reflections on the appropriation of scientific knowledge in non-formal education. This topic is of particular interest to professionals that are mindful of the relation between science and society due to three issues. Firstly, it propitiates educators to review the theories of learning that underlie their practices. Second, it provides museum experts with a scientific approximation to the proper knowledge. Finally, it encourages researchers to contemplate the social distribution of scientific knowledge and the role of museums in the national context. 


\section{Keywords}

scientific knowledge appropriation; cyber museum; non-formal education; museums and proper knowledge; Mexico

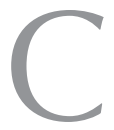
omo su título lo indica: Contextos educativos no formales: el museo y la apropiación del conocimiento científico (García y Espinosa 2015), es un libro que examina la apropiación del conocimiento en contextos educativos no formales y, en particular, cuán valiosamente el museo incide en el bienestar individual y social.

La valía de esta producción académica radica, por un lado, en que invita a evaluar, desde la perspectiva de la apropiación del conocimiento, las prácticas museológicas vigentes en México, y, por el otro, en que señala que es pertinente indagar las condiciones en que se logra la distribución social del conocimiento de tipos tanto intuitivo como científico (Crovi 2006:156-159), así como la función que juegan las instituciones públicas para su divulgación y apropiación.

Los ocho autores compendiados comparten la premisa de que apropiarse de un objeto de conocimiento significa tomar algo para sí por derecho propio mediante un proceso que modifica estructuras cognitivas para solucionar problemáticas que apelan a una cuestión vital para el individuo o el colectivo (Peñaloza y Méndez 2015:96-97). Es interesante, entonces, la presencia de dos grados de apropiación: se busca que los usuarios de museos la asuman respecto del conocimiento científico que se exhibe, en tanto que, en sí, el libro representa una una apropiación científica, tanto del corpus museológico como de los procesos cognitivos presentes en los contextos educativos no formales, cuyo fin es generar condiciones óptimas para influir en los usuarios de los espacios expositivos.

Los autores plantean una trama multidisciplinar —la cual involucra la arquitectura, la comunicación, el diseño, la educación, la filosofía de la ciencia y la sociología-que parte de una estructura binaria para examinar su objeto: el museo como contexto de educación no formal. El libro consta de seis capítulos. Los dos primeros dan referencias teóricas sobre el desarrollo del museo (Segurajáuregui 2015:17-26; Contreras 2015:27-48), mientras que los dos intermedios presentan evidencias empíricas de investigaciones en curso (González de Cossío 2015:4970; García y Espinosa 2015:71-94) y los finales abordan reflexiones epistemológicas (Peñaloza y Méndez 2015:95-126; Casanueva y Méndez 2015:127-148). En los siguientes párrafos se indican sus aportaciones y las posibles relaciones conceptuales entre ellos.

La publicación inicia con una apretada síntesis histórica del surgimiento del museo en el coleccionismo (Segurajáuregui 2015:17-26), que pone el énfasis en la importancia de su función educativa desde mediados del siglo XX, con inclusión de los cambios recientes, así como las nuevas tipologías museísticas, los programas arquitectónicos y el papel de las exposiciones temporales. En el entendido de que se trata de un resumen para todo tipo de lectores, es plausible que la bibliografía - mayoritariamente de investigadores españoles- sea de fácil acceso; sin embargo, no se advierte un eje narrativo ni un referente que sustente los comentarios finales, como tampoco en relación con la crítica a la venta de objetos asociada con las colecciones (Segurajáuregui 2015:26).

El segundo capítulo presenta la noción de "museo como institución", y una revisión a la temporalidad y la espacialidad museísticas (Contreras 2015:27-48), con lo que, tomando en cuenta el imaginario social del visitante, se argumentan las particularidades del cibermuseo. La autora examina la noción del "museo virtual" como antecedente de éste, que va más allá de la función de difusión de las actividades del museo físico, para lo que propone considerar tres dimensiones relacionales: la intrasubjetiva, la intersubjetiva y la transubjetiva, que influirán de manera eficaz en el vínculo museo-usuario.

Este texto no refiere explícitamente la apropiación del conocimiento, sino su función en el conjunto: la apertura hacia discusiones actuales, como el surgimiento de la cibermuseología. ${ }^{1}$

En el tercer capítulo de su obra, González de Cossío (2015:49-70) describe el diseño y la evaluación de una exhibición itinerante sobre la movilidad social y la educación, titulada Imagina tu futuro (CEEY 20132017), que, auspiciada por una fundación privada y enfocada en jóvenes, aporta una metodología para evaluar en qué medida influye en las actitudes de los usuarios, identifica los procesos cognitivos deseables en cada segmento expositivo mediante retóricas a escala lógica y emocional y, en otro nivel, apela a la credibilidad. En este apartado del libro se advierten dos ausencias; en primer lugar, no hay referencia a la relación entre los museos y el sistema educativo mexicano, y, en segundo, la evaluación no toma en cuenta que la exposición Imagina tu futuro se presenta en contextos educativos formales, con uso de recursos ya bien asimilados, tales como la visita grupal, la presencia del maestro y el apoyo de guías.

En el cuarto capítulo, el espacio museístico se conceptualiza como espacio social (García y Espinosa 2015:71-94); en él las autoras derivan de ese hecho la necesidad de examinar la apropiación del espacio a través de las acciones, la construcción de significados, las creencias o las características propias de un lugar.

Una lectura sugerente es el parangón entre la postura del diseño

\footnotetext{
${ }^{1}$ Para un estado del arte actualizado respecto del objeto de estudio de la cibermuseología, sus alcances y aportaciones, y la comprensión de las dimensiones digitales del museo, véase Anna Leshchenko (2015).
} 
que, en el capítulo precedente, asume como principio usar conceptos y formas de presentación familiares a los usuarios y las prácticas que realiza una persona con un determinado habitus, que le permite decodificar el museo y apropiarse plenamente de sus contenidos y formas. De esta manera, las autoras retoman el planteamiento de Pierre Bourdieu sobre el museo como capital cultural objetivado y refrendan la importancia del habitus para comprender las prácticas culturales. Este capítulo distingue entre los conocimientos específicos apropiados mediante una exposición y el nivel de apropiación tanto del museo en sí mismo como de sus códigos, lo cual, sin embargo, no se explora en su metodología.

Los capítulos restantes emprenden reflexiones de tipo epistemológico. El quinto, escrito por Peñaloza y Méndez (2015:95-126), define la apropiación del conocimiento y examina las metáforas implícitas en las teorías del aprendizaje: adquisición (mente como recipiente), construcción (mente como generadora) y participación (mente como práctica cultural). Los autores proponen condiciones para que ocurran tanto la primera: una base común de la apropiación del conocimiento y el cuestionamiento de las premisas existentes, como las siguientes: la disponibilidad de modelos teóricos y las aplicaciones sobre el asunto de interés; la experiencia propia que modifica dichos modelos; la consolidación de conocimientos previos, y la emergencia de nuevas ideas.

El texto examina tres procesos cognitivos presentes en dicha apropiación: la metáfora, la enacción y la teoría de los espacios conceptuales, y cierra con una breve descripción de la tipología de los espacios de aprendizaje (Peñaloza y Méndez 2015:115-121).

El capítulo ulterior centra la atención sobre el conocimiento como sistema de distribución y el concepto de "infomorfismo" como relación o mapeo entre entidades (Casanueva y Méndez 2015:127-148), para ar- gumentar que el conocimiento científico se conforma por una red que abarca desde saberes teóricos, hasta instrumentos y configuraciones de datos: si opera como red de tipo híbrido type/token, se lo puede representar en sistemas conceptuales y relacionales y comprender lo que significa apropiarse de un concepto: "simplemente estar en posesión de sus contenidos" (Casanueva y Méndez 2015:141), es decir, poseer, en diversos grados, los contenidos de esa red multidimensional.

En lo individual, significa poseer la teoría y el manejo de procedimientos y técnicas, mientras que la apropiación social "implica no sólo hacerse del dato o la información, sino también de los patrones de inferencia usuales en el quehacer de esa ciencia" (Wagner 2007, citado en Casanueva y Méndez 2015:143).

En suma, los distintos intereses de investigación convergen atinadamente en esta publicación, un conjunto coherente en torno de la apropiación del conocimiento científico y de la relevancia, en nuestra sociedad, de los espacios de educación no formal para lograr su divulgación y apropiación. Por momentos, su brevedad (no supera las 150 páginas) marca un ritmo apresurado, no obstante lo cual las proposiciones manifiestan, en general, rigor académico, y algunos textos exponen oportunos esquemas, tablas y gráficas. El lector atento identificará, en función de su interés personal, ciertos temas interesantes en las relaciones intertextuales, así como las especialidades que se suman al quehacer museológico (por ejemplo, el diseño de información o la gestión del conocimiento), los matices comunicativos para que el usuario acepte al museo como autoridad, o para afectar sus emociones sin desvelar la agenda de la institución.

Por otra parte, se pone en falta una mayor referencia a estudios ejemplares, ya sea en exposiciones de divulgación científica (cfr. Castellanos 2008) o de arte (cfr. Rosas Mantecón 2008), así como un anclaje más sólido para llevar a la práctica alguna herramienta o concepto procedente de las reflexiones epistémicas.

Al inicio de la reseña mencioné que la distribución social del conocimiento permite alcanzar mayor bienestar, lo que se emparenta con la ciudadanía que se ejerce: el hombre común, guiado por sus opiniones; el experto, sumamente especializado en una rama del conocimiento, y el ciudadano bien informado, que no busca el grado de análisis del anterior, pero, de acuerdo con sus intereses, sí demanda información relevante (Crovi 2008:157). Las instituciones -como la universidad o el museo- ¿tendrán la capacidad de gestionar esa movilidad de saberes si se enfocan en la divulgación de asuntos de interés público y de gran relevancia para distintos sectores de la población, tomando en cuenta algunos de los parámetros propuestos en este libro?

En el caso del museo, esto exige transitar de un enfoque centrado en la operación museística a otro orientado al usuario. La apropiación recrea no sólo lo que conocemos, sino cómo lo conocemos; así, el museo podría resultar insustituible justamente por ello, puesto que nos coloca frente a operaciones de recontextualización de lo real que exigen una vigilancia epistémica singular.

\section{Referencias}

Casanueva López, Mario y Diego Méndez Granados

2015 "Apropiación del conocimiento empírico", en Caridad García Hernández y Margarita Espinosa Meneses (coords.), Contextos educativos no formales: el museo y la apropiación del conocimiento científico, México, Universidad Autónoma MetropolitanaUnidad Cuajimalpa, 127-148.

Castellanos Pineda, Patricia

2008 Los museos de ciencias y el consumo cultural. Una mirada desde la comunicación, Barcelona, Universitat Oberta de Catalunya.

CEEY

2013-2017 Imagina tu futuro, Exposición itinerante, México, Centro de Es- 
tudios Espinosa Yglesias (CEEY), documento electrónico [página web] disponible en [http://www.imaginatufuturo.org.mx/ exposición], consultado en noviembre de 2017.

Contreras García, María del Carmen

2015 "El imaginario social del museo al cibermuseo", en Caridad García Hernández y Margarita Espinosa Meneses (coords.), Contextos educativos no formales: el museo y la apropiación del conocimiento científico, México, Universidad Autónoma Metropolitana-Unidad Cuajimalpa, 27-48.

Crovi Druetta, Delia

2006 "Universidad y sociedad del conocimiento", en Educar en la era de las redes, México, Universidad Nacional Autónoma de México, 153-179.

García Hernández, Caridad y Margarita Espinosa Meneses 2015 "Espacio, cuerpo y apropiación de conocimiento en los museos", en Caridad García Hernández y Margarita Espinosa Meneses (coords.), Contextos educativos no formales: el museo y la apropiación del conocimiento científico, México, Universidad Autónoma Metropolitana-Unidad Cuajimalpa, 71-94.

González de Cossío, Rosenzweig

2015 "La apropiación del conocimiento para influir en las actitudes. De los datos a la experiencia en una exposición", en Caridad García Hernández y Margarita Espinosa Meneses (coords.), Contextos educativos no formales: el museo y la apropiación del conocimiento científico, México, Universidad Autónoma Metropolitana-Unidad Cuajimalpa, 49-70.

Leshchenko, Anna

2015 "Digital dimensions of the museum: defining Cybermuseology's subjetc of study", Icofom Study Series, 43:237-241, documento electrónico [pdf] disponible en [https://www.researchgate.net/publication/311065086_ Digital_Dimensions_of_the_Museum_Defining_ Cybermuseology\%27s_Subject_of_Study], consultado en noviembre de 2017.

Peñaloza Castro, Eduardo y Diego Méndez Granados 2015 "La apropiación del conocimiento en comunicación y educación para la ciencia", en Caridad García Hernández y Margarita Espinosa Meneses (coords.), Contextos educati- vos no formales: el museo y la apropiación del conocimiento científico, México, Universidad Autónoma MetropolitanaUnidad Cuajimalpa, 95-126.

Rosas Mantecón, Ana y Graciela Schmilchuck 2008 “Comunicar o someter? Evaluación de dispositivos de interpretación de la exposición El cuerpo aludido (Museo Nacional de Arte, 1999)", Cuicuilco, 44:59-86.

Segurajáuregui Álvarez, Elena

2015 "Coleccionismo y el origen del museo", en Caridad García Hernández y Margarita Espinosa Meneses (coords.), Contextos educativos no formales: el museo y la apropiación del conocimiento científico, México, Universidad Autónoma Metropolitana-Unidad Cuajimalpa, 17-26.

\section{Síntesis curricular del/los autor/es}

\author{
Norma Angélica Avila Meléndez \\ Instituto de Educación Media Superior del Distrito Federal (IEMS-DF), \\ México \\ norma.avila@iems.edu.mx
}

Diseñadora gráfica (Universidad Nacional Autónoma de México [UNAM]) y maestra en museología (Escuela Nacional de Conservación, Restauración y Museografía, Instituto Nacional de Antropología e Historia [ENCRyM-INAH], México). Ha colaborado en el Museo Nacional de Culturas Populares de la Secretaría de Cultura (MNCP-SC, México), así como en la capacitación y la formación de organizaciones a través de los programas nacionales de Museos Comunitarios y de Espacios Comunitarios, ambos impulsados por la Coordinación Nacional de Museos y Exposiciones (CNME-INAH, México). Es miembro del ICOFOM (International Committee for Museology/International Council of Museums [ICOM]; Comité Internacional para la Museología/ Consejo Internacional de Museos) y ha presentado ponencias y publicaciones en diversos foros nacionales e internacionales. Es docente-investigadora en el Instituto de Educación Media Superior del Distrito Federal (IEMS-DF, México), donde realiza su investigación doctoral sobre prácticas de arte colaborativo (Facultad de Artes y Diseño [FAD-UNAM], México).

Postulado/Submitted: 21.03 .2017

Aceptado/Accepted: 07.12.2017

Publicado/Published: 28.02.2018

Intervención, Revista Internacional de Conservación, Restauración y Museología, año 9, número 17, enero-junio de 2018, se terminó de imprimir el 28 de febrero de 2018, en los talleres de Calle

Ciudad de México.

La edición consta de 1000 ejemplares, impresos en papel cultural ahuesado de 90 gr para interiores, y en couché de 250 gr para los forros. 